\title{
MODELAGEM MATEMÁTICA DE OTIMIZAÇÃO APLICADA AO PLANEJAMENTO DO PLANTIO DE CANA-DE-AÇÚCAR E CANA-ENERGIA
}

CARMO, Carlos Roberto Souza ${ }^{1}$

\author{
Recebido em: 2021.03 .03
}

Aprovado em: 2021.04.27

ISSUE DOI: $10.3738 / 1982.2278 .3900$

\begin{abstract}
RESUMO: Esta investigação científica teve por objetivo propor uma modelagem matemática de otimização voltada para o apoio à tomada de decisão referente à minimização dos custos de produção da cana-de-açúcar e da canaenergia, a partir do planejamento referente à escolha das variedades de cultivares a serem plantados em determinada área de cultivo, e ainda, visando identificar dimensionamento máximo dos talhões a serem ocupados por cada uma dessas variedades, a fim a garantir: a diversificação varietal; a ocupação total da área destinada ao plantio da cana; e, atender às demandas de sacarose e fibra de empreendimentos sucroenergéticos. A partir de técnicas baseadas em problemas de programação linear, o modelo proposto foi implementado computacionalmente em três cenários distintos, com simulações de diferentes níveis de concentração varietal, e mostrou-se eficiente não só no cumprimento das suas premissas básicas, mas, também mostrou-se especialmente eficiente ao proporcionar informações capazes de permitir apurar o custo do processo de diversificação varietal propriamente dito.
\end{abstract}

Palavras-Chave: Diversificação. Risco. Métodos quantitativos aplicados.

\section{MATHEMATICAL MODELING OF OPTIMIZATION APPLIED TO PLANNING OF SUGARCANE AND ENERGY CANE PLANTING}

SUMMARY: This scientific investigation aimed to propose a mathematical optimization model aimed at supporting decision making regarding the minimization of the production costs of sugar cane and energy cane, based on the planning regarding the choice of varieties of cultivars to be planted in a certain cultivation area, and also, aiming to identify the dimensioning of the plots to be occupied by each one of these varieties, in order to guarantee: the varietal diversification; the total occupation of the area destined to the planting of sugarcane and energy cane; and, to meet the sucrose and fiber demands of sugar-energy projects. Based on techniques based on linear programming problems, the proposed model was implemented computationally in three different scenarios, with simulations of different levels of varietal concentration, and proved to be efficient not only in fulfilling its basic premises, but also proved to be effective. it is especially efficient in providing information capable of determining the cost of the varietal diversification process itself.

Keywords: Diversification. Risk. Quantitative methods applied.

\section{INTRODUÇÃO}

A cana-de-açúcar é uma das culturas mais relevantes no agronegócio nacional. Além da expressiva produção nacional de etanol, o Brasil é um dos maiores produtores de açúcar no mundo, e, a energia elétrica produzida a partir de biomassa de cana-de-açúcar é responsável por mais de $15 \%$ da matriz energética brasileira (UNICA, 2016).

A área cultivada de cana-de-açúcar é uma das três maiores áreas cultivadas em todo território brasileiro, e o total dos investimentos realizados nos negócios da cadeia produtiva sucroenergética são cada vez maiores (LORIZOLA; CAPITANI, 2018).

Devido à importância da cultura da cana-de-açúcar para o agronegócio brasileiro, alguns aspectos se tornam relevantes em relação ao planejamento e à tomada de decisão voltados para o

\footnotetext{
${ }^{1}$ ORCID- https://orcid.org/0000-0002-3806-9228. Professor adjunto da Faculdade de Ciências Contábeis da Universidade Federal de Uberlândia (FACIC-UFU).
} 
seu plantio. E, esse tipo de decisão ganha relevância não só em função da representatividade dessa cultura para a economia nacional, mas, especialmente pelo fato de se tratar de uma cultura que, uma vez plantada, permanece vinculada ao solo por cerca de três a quatro.

Nesse sentido, o processo de planejamento e escolha de cultivares destinados ao plantio e cultivo dessa cultura merece especial atenção, quer seja por aspectos relacionados à produtividade das variedades a serem cultivadas, quer seja pela necessidade de diversificação varietal, cuja ausência pode trazer riscos e prejuízos para os empreendedores desse tipo de atividade agrícola.

A diversificação varietal diz respeito à escolha dos diferentes tipos de cultivares de cana a serem plantados a fim de evitar a concentração de uma mesma variedade em determinada área produtiva, constituindo-se em uma estratégia destinada a proporcionar segurança biológica, podendo evitar que pragas ou doenças sejam capazes de atingir uma grande parte do canavial, comprometendo severamente toda a produção de determinada região (BRASIL, 2016).

Dados do último censo varietal indicam que mais da metade da área ocupada pela cultura da cana-de-açúcar na região centro-sul $(54,8 \%)$ está concentrada em apenas cinco tipos de cultivares, ou seja: a variedade RB867515 que ocupa 27,1\% da área cultivada na região centrosul; a variedade RB966928 que cupa 9,1\%; a SP81-3250 que ocupa 7,5\%, a RB92579 que ocupa 6,1\%; e, a variedade RB855453 que ocupa 5,0\% (BRAGA JUNIOR et al, 2017).

Tais índices podem ser considerados críticos e tornam a cultura suscetível ao ataque de doenças, pragas ou plantas daninhas, o que pode levar a prejuízos muito significativos para o setor (TONIELO et al, 2018).

Sem perder de vista a preocupação com a diversificação varietal, o processo de planejamento relacionado ao plantio da cana também deve levar em conta os fatores relacionados à qualidade produtiva da cultura, isso é, à quantidade de sacarose presente na cana-de-açúcar e, ainda, à respectiva quantidade de fibra produzida, entre outros fatores.

A sacarose representa a matéria-prima básica destinada à produção de açúcar e etanol, e a fibra presente na cana constitui-se na matéria seca utilizada na queima para a produção de calor e energia elétrica a partir do processo de cogeração energética.

Além desses aspectos técnico-produtivos, destaca-se o fator custo, que é especialmente relevante em qualquer atividade econômica. Pois, o relacionar a temática dos custos ao processo de planejamento, a literatura disponível indica que a partir de $70 \%$ dos custos são determinados na fase do planejamento (ANSARI; BELL, 1997; MARTYNIUK; ZABLOCKA, 1998), podendo superar 90\% do seu total (COOPER; SLAGMULDER, 1997; SAKURAI, 1997). 
Nesse contexto, esta investigação teve por objetivo propor uma modelagem matemática de otimização, baseada em programação linear, voltada para o apoio à tomada de decisão referente à escolha de quais variedades de cana-de-açúcar e cana-energia plantar, de maneira a atender as especificações técnico-produtivas inerentes aos empreendimentos sucroenergéticos (demandas por sacarose e fibra), garantir os níveis mínimos de diversificação varietal, e ainda, minimizar os respectivos custos de produção.

Uma vez que a pesquisa operacional pode auxiliar cientificamente a tomada de decisões envolvendo a busca pela solução de problemas relacionados à alocação de recursos em cenários restritivos (ARENALES et al., 2015), e dentre suas ferramentas encontra-se a pesquisa e proposição de modelos matemáticos de otimização, este estudo de natureza científica se justifica pela possibilidade de fornecer subsídios para a quantificação prévia de gastos e efeitos decorrentes do planejamento de ações essenciais para o sucesso de empreendimentos agrícolas produtores de cana-de-açúcar e cana-energia. Além disso, deve-se considerar o fato de que os experimentos matemático-computacionais são valiosas ferramentas de produção de informações necessárias ao planejamento e à gestão, cujos custos são bem menores que os custos dos experimentos agrícolas convencionais (DEJENIE, 2019).

\section{REFERENCIAL TEÓRICO}

Estão seção foi dividida em duas outras seções secundárias a fim de viabilizar a compreensão da temática relacionada à complexidade envolvida no processo de escolha de variedades de cana para plantio, bem como, os respectivos parâmetros de decisão; e, em uma segunda parte, apresentar um panorama geral acerca do perfil das pesquisas científicas voltadas para o processo de modelagem matemática de otimização aplicada à cultura da cana-de-açúcar.

\subsection{Principais Parâmetros Relacionados à Escolha da Cana para Plantio}

Isler et al (2014) destacam que no processo de escolha das variedades de cana para plantio dois fatores merecem destaque: Pol e fibras. A Pol representa a quantidade de sacarose aparente contida no caldo da cana-de-açúcar, e as fibras representam a matéria sólida presente na cana e que, normalmente, é queimada após a extração do caldo de forma a produzir calor, vapor e energia para suprir as demandas da usina e permitir a comercialização dos excedentes de energéticos (ISLER et al, 2014).

Para Vian (2018) a quantidade média de Pol deve ser superior a 14\% na cana-de-açúcar colhida, e o percentual de fibras deve se situar entre $11 \%$ e $13 \%$. Nervis (2015) considera que a cana-de-açúcar padrão deve apresentar Pol em torno de $12 \%$ e teor de fibras de $8 \%$ a $14 \%$. Matsuoka (2017) afirma que, de uma forma muito simplificada, se $70 \%$ do peso da cana-de- 
açúcar é formado por água, os 30\% restantes são divididos entre sacarose e fibras em uma proporção ideal de $16 \%$ de Pol e $14 \%$ de fibras

Ao analisar os fatores que contribuíram para a queda dos níveis de açúcares totais recuperáveis ao longo das safras de 1984 e 2015, Cherubin (2015) destaca que a Pol da cana-deaçúcar variou de um mínimo de 11,6\% até um máximo de 16\% ao longo dessa década, perfazendo uma média de 12,90.

Para Caversan (2017), a cana-de-açúcar deve apresentar um teor médio de fibras de 12,9\% e a cana-energia, caracterizada por apresentar maiores níveis de fibra do que sacarose, pode apresentar um teor de fibras em torno de $17,9 \%$.

Caversan (2017) afirma que, apesar de também produzir sacarose, a cana-energia possui como principal característica a maior produtividade de massa seca, o que faz com ela seja muito utilizada na produção de energia e etanol de segunda geração.

Dessa forma, percebe-se que existe uma amplitude bem elástica em relação aos percentuais mínimos e máximos a serem considerados como parâmetros esperados em termos de produtividade de sacarose aparente no caldo da cana (Pol) e de fibras. Ou seja, observam-se valores que vão de um mínimo de 11,6\% até um máximo 16\% (CHERUBIN, 2015) para a Pol, e um mínimo de $8 \%$ até um máximo de 14\% para a fibra (NERVIS, 2015), podendo chegar até a 17,90\% no caso da cana-energia (CAVERSAN, 2017).

Acerca da diversificação varietal, enquanto prática a ser adotada para proporcionar a devida segurança biológica à cultura da cana, Raizer, Braga Júnior e Selegato (2013) ponderam que a área ocupada por cada variedade de cultivar de cana não deve ultrapassar um grau de concentração em um só variedade superior a $15 \%$ da área plantada total, conforme recomendações do Centro de Tecnologia Canavieira (CTC). Sendo que, tal medida tem por finalidade evitar que a ocorrência de pragas e/u doenças sejam capazes de comprometer toda a produção dos canaviais (BRASIL, 2016).

Contudo, pode-se presumir que uma mesma unidade produtora de cana-de-açúcar e/ou cana-energia utilizará aquela quantidade de variedades de cana que lhe for conveniente, porém, sem deixar de buscar a maior diversificação varietal possível e viável economicamente, e ainda, sem perder de vista que o ideal é que toda a sua área cultivável seja ocupada, além do planejamento voltado para o atendimento das demandas de matéria-prima do setor sucroenergético, portanto, níveis mais elevados possíveis de sacarose e fibra.

Independentemente da relevância e dos montantes de recursos financeiros envolvidos na cadeia de negócios pertencentes ao setor sucroenergético nacional, a produção desse segmento 
econômico pode ser considerada um conjunto de commodities cujo preço é definido pelo mercado (TOMAZELA; CAMPOS; DANIEL, 2015).

Devido a isso, inexiste a possibilidade das empresas sucroenergéticas ajustarem seus preços de venda à revelia das condições impostas pelo mercado no qual encontram-se inseridas. Logo, resta-lhes concentrar esforços no planejamento e controle dos seus processos, produtivos e comerciais, a fim de implementar, sempre que possível, uma gestão eficaz de custos com vistas a melhores resultados econômicos (TOMAZELA; CAMPOS; DANIEL, 2015).

Ramos (2010) realizou uma investigação científica na qual foi proposto um modelo matemático de otimização multi-objetivo em que buscou-se minimizar custos de produção e maximizar a produção de energia, açúcar e etanol de unidades de negócio sucroenergéticas. Durante sua investigação, Ramos (2010) observou que para uma área produtiva de 315,81 hectares (ha) o custo de preparo do solo poderia atingir cerca de $\mathrm{R} \$ 163.763,28$; para o plantio seriam gastos $\mathrm{R} \$ 897.115,15$; a colheita poderia custar $\mathrm{R} \$ 690.461,70$; e, para a coleta e aproveitamento do palhiço gerado pela colheita mecanizada seriam gastos $\mathrm{R} \$ 94.362,92$. Tudo isso totalizaria um montante de $\mathrm{R} \$ 1.845 .703,05$ em uma única safra, perfazendo um custo unitário de $\mathrm{R} \$ 5.844,35 \mathrm{ha}^{-1}\left(\mathrm{R} \$ 1.845 .703,05 / 315,81 \mathrm{ha}=\mathrm{R} \$ 5.844,35 \mathrm{ha}^{-1}\right)$.

Contudo, deve-se levar em conta que de $70 \%$ a $90 \%$ desses custos podem ser definidos na fase de planejamento de um negócio (ANSARI; BELL, 1997; MARTYNIUK; ZABLOCKA, 1998; COOPER; SLAGMULDER, 1997; SAKURAI, 1997). Nesse sentido, o planejamento voltado para o cultivo da cana pode ser direcionado, em grande parte, a partir do processo de escolha das variedades de cana a serem cultivadas.

Assim, ao considerar a complexidade das atividades necessárias à implementação desse tipo de atividade econômico-produtiva, e ainda, o grande número de variáveis envolvidas, percebe-se que o planejamento relacionado à escolha de variedades para plantio é uma atividade crítica para o sucesso desse tipo de empreendimento agrícola. Contudo, conforme observado por Isler et al (2014), diante da diversidade e da natureza das variáveis envolvidas, o planejamento produtivo no setor sucroenergético não é uma tarefa fácil e, para tanto, os gestores podem lançar mão de recursos relacionados à aplicação de modelagens matemáticas otimização voltadas para a minimização de custos e elevação da produtividade, conforme proposto por esta investigação.

\subsection{Modelagem Matemática de Otimização Aplicada à Cultura da Cana-de-açúcar}

Dada a relevância do setor sucroenergético para a economia nacional e diante da intensificação de suas atividades produtivas, a complexidade dos problemas relacionados à tomada de decisão neste segmento tem aumentado consideravelmente, o que o torna esse propício à aplicação das técnicas de otimização matemática (CARMO, 2020; NERVIS et al, 2017). 
Ao realizarem um extenso levantamento acerca das pesquisas científicas voltadas para a utilização de modelagens matemáticas no processo de otimização da colheita de cana-de-açúcar, Doriguel, Crusciol e Florentino (2017) identificaram um total de 34 estudos realizados entre 1977 e 2017.

Uma análise detalhada dos estudos identificados por Doriguel, Crusciol e Florentino (2017) revelou que, em sua maioria, essas pesquisas buscaram minimizar os custos do processo de colheita e/ou elevar a produtividade de sacarose da cultura da cana, a partir da identificação do momento ideal da colheita.

A partir de 2014, pôde-se observar a realização de estudos utilizando o processo de modelagem matemática de otimização aplicada a cultura da cana-de-açúcar que passaram a contemplar também variáveis agronômicas propriamente ditas (SILVA; MARINS, 2014 apud DORIGUEL, CRUSCIOL, FLORENTINO, 2017; SILVA; MARINS; DIAS, 2015 apud DORIGUEL, CRUSCIOL, FLORENTINO, 2017; RAMOS et al., 2016, apud DORIGUEL, CRUSCIOL, FLORENTINO, 2017).

A partir de 2017, pôde-se observar certa diversificação tanto dos métodos de modelagem propriamente ditos quanto das variáveis abordadas no processo de otimização, apesar de ainda existir a predominância do foco nas atividades de colheita da cana-de-açúcar. Nesse contexto, destacam-se as pesquisas realizadas por Sungnul et al. (2018), Caixeta-Filho e Miyashita (2018), Patcharamethanon e Sudtachat (2018) e Florentino et al. (2018) e Carmo (2020).

Sungnul et al. (2018) utilizaram uma modelagem matemática de otimização bi-objetivo para minimizar o custo de colheita da cana-de-açúcar, e ainda, maximizar a receita de venda da cana colhida no mercado tailandês. O modelo matemático pesquisado por Sungnul et al. (2018) permitiu implementar ações para redução dos custos de colheita mediante a identificação do tempo ótimo de colheita da cana-de-açúcar na Tailândia, ao longo das safras de 2012/2013, 2013/2014, 2014/2015 e 2015/2016.

Caixeta-Filho e Miyashita (2018) pesquisaram e propuseram um modelo matemático baseado em programação linear inteira mista visando a maximização da receita de venda do açúcar a partir da otimização da colheita da cana, com base em parâmetros restritivos relacionados à capacidade de processamento e à parcela mínima da área cultivada ser colhida. Nesse sentido, Caixeta-Filho e Miyashita (2018) propuseram uma escala horária para a realização da colheita que permitia ampliar a proporção da área colhida.

Em sua investigação, Patcharamethanon e Sudtachat (2018) utilizaram um modelo matemático de otimização para a minimizar o custo com transporte da cana-de-açúcar colhida, mediante a demarcação de setores de colheita e o processo rodízio de caminhões de seis e dez 
rodas. Patcharamethanon e Sudtachat (2018) avaliaram que os fatores críticos para os custos do transporte da cana-de-açúcar colhida estavam relacionados aos processos de manobras de corte, carga e descarga da cana nos caminhões.

Florentino et al. (2018) também focaram sua pesquisa no processo de colheita e nos respectivos custos, e ainda, na maximização da sacarose, porém, realizaram a diversificação do processo de modelagem matemática de otimização ao empregar técnicas aproximativas, e ainda, por se preocuparem com variáveis relacionadas ao processo de cultivo da cana-de-açúcar propriamente dito. Ao testar computacionalmente um algoritmo genético, comparativamente a um método de otimização exato, Florentino et al. (2018) concluíram que para a solução de problemas de fazendas de médio e grande porte, os métodos aproximativos baseados em heurística (técnicas aproximativas) são mais recomendados, ao passo que os métodos exatos se aplicam melhor a problemas de otimização de fazendas de pequeno porte.

Utilizando o método de Programação por Metas Ponderada (Weighted Goal Programming), Carmo (2020) propôs e testou um modelo matemático de otimização cujo objetivo foi minimizar os gastos com a aquisição e aplicação de agentes fisiológicos indutores de maturação, e ainda, buscou garantir o atendimento da demanda de cana-de-açúcar de empresas sucroenerméticas, ao longo de toda a safra. Além de atingir seu objetivo central, a modelagem matemática proposta por Carmo (2020) mostrou-se capaz de fornecer informações voltadas para o suporte da tomada de decisão relacionada também à compra de maturadores, ao respectivo orçamento, bem como, à identificação de cada talhão para aplicação de diversos tipos de agentes fisiológicos de maturação.

Dessa forma, pode-se observar que, mesmo diante da diversificação das metodologias utilizadas no processo de modelagem matemática de otimização e da existência de certa preocupação com a utilização de algumas das variáveis relacionadas ao processo de cultivo propriamente dito, e até mesmo, diante da utilização de variáveis relacionadas ao processo de comercialização da cana-de-açúcar (vendas e suas receitas, por exemplo), a maioria dos estudos sobre modelagens matemáticas de otimização aplicadas a cultura da cana ainda estão voltados para o processo de colheita, em detrimento ao estudo de variáveis relacionadas com o cultivo em geral e, em especial, ao início do ciclo produtivo da cana, ou seja, o planejamento do plantio.

Nesse contexto, a presente pesquisa pode representar uma contribuição de grande utilidade, uma vez que ela se concentra no processo de identificação das variedades de cana-deaçúcar e cana-energia a serem plantadas e no dimensionamento máximo de cada talhão, para que se promova tanto a minimização dos riscos da cultura a partir da a diversificação varietal, quanto a minimização dos respetivos custos de produção, sem deixar de atender as especificações 
técnico-produtiva inerentes à demanda de matéria-prima dos empreendimentos sucroenergéticos (sacarose e fibra).

\section{MATERIAL E MÉTODO}

O modelo proposto nesta pesquisa foi desenvolvido a partir de técnicas baseadas em problemas de programação linear (PPL), com o objetivo de minimizar os custos da produção da cana-de-açúcar e da cana-energia, a partir do planejamento referente à escolha das variedades de cultivares a serem plantados em determinada área de cultivo, e ainda, visando identificar o dimensionamento máximo dos talhões a serem ocupados por cada uma dessas variedades, a fim a garantir: a diversificação varietal; a ocupação total da área destinada ao plantio da cana; e, atender às demandas de sacarose e fibra de empreendimentos sucroenergéticos.

O referido modelo matemático de otimização tem a sua função objetivo descrita pela Equação 1, que está sujeita às restrições representadas pelas Equações 2 até 8, conforme as variáveis, índices e parâmetros detalhados no Quadro 1.

Minimize $\quad \sum_{i=1}^{m} C_{i} X_{i}+\sum_{j=1}^{n} C_{i} X_{j}$

Sujeito a:

$$
\begin{aligned}
& X_{i} \leq G c(A a+A e) \quad \forall i \\
& X_{j} \leq G c(A a+A e) \quad \forall j \\
& \sum_{i=1}^{m} X_{i}=A a \\
& \sum_{j=1}^{n} X_{j}=A e \\
& \sum_{i=1}^{m} X_{i} \operatorname{Prod}_{i} \operatorname{Pol}_{i}+\sum_{j=1}^{n} X_{i} \operatorname{Prod}_{j} \text { Pol }_{j} \geq D_{\text {Sacarose }} \\
& \sum_{i=1}^{m} X_{i} \operatorname{Prod}_{i} \text { Fib }_{i} \geq D_{\text {Fibra }}+\sum_{j=1}^{n} X_{j} \text { Prod }_{j} \text { Fib }_{j} \geq D_{\text {Fibra }} \\
& X_{i}, X_{j} \geq 0
\end{aligned}
$$


Quadro 1 - Descrição dos variáveis, índices e parâmetros do modelo proposto

\begin{tabular}{|c|c|l|}
\hline Legenda & Categoria & \multicolumn{1}{c|}{ Descrição } \\
\hline$i=i, \ldots . m$ & índice & índice representativo de cada variedade de cana-de-açúcar a ser plantada. \\
\hline$j=j, \ldots n$ & índice & índice representativo de cada variedade de cana-energia a ser plantada. \\
\hline$X$ & $\begin{array}{c}\text { variável de } \\
\text { decisão }\end{array}$ & área máxima (ha) a ser destinada ao plantio de cada variedade de cana. \\
\hline$C$ & parâmetro & Custo por hectare $\left(\mathrm{R} \$\right.$ ha $\left.^{-1}\right)$ de cana plantada e colhida, com coleta de palhiço. \\
\hline$G c$ & parâmetro & $\begin{array}{l}\text { Grau de concentração percentual máximo admitido para ocupação da área total por } \\
\text { cada variedade de cana a ser plantada, garantindo a diversificação varietal. }\end{array}$ \\
\hline$A a$ & parâmetro & parcela da área total destinada ao plantio de cana-de-açúcar $($ ha). \\
\hline$A e$ & parâmetro & parcela da área total destinada ao plantio de cana-energia (ha). \\
\hline Prod & parâmetro & produtividade prevista, em toneladas (t), para cada hectare (ha) de cana colhida. \\
\hline Pol & parâmetro & percentual de sacarose presente no caldo de cada tonelada (t) de cana colhida. \\
\hline Fib & parâmetro & percentual de fibra presente em cada tonelada (t) de cana colhida. \\
\hline$D_{\text {sacarose }}$ & parâmetro & demanda total de sacarose prevista para toda a safra, em toneladas (t). \\
\hline$D_{\text {fibra }}$ & parâmetro & demanda total de fibras prevista para toda a safra, em toneladas $(\mathrm{t})$. \\
\hline
\end{tabular}

Fonte: elaborado pelo autor, a partir dos dados pesquisa.

Enquanto função objetivo do modelo proposto por esta pesquisa, a Equação 1 tem por finalidade garantir a minimização do custo total da produção de cana-de-açúcar $(i)$ e o custo total da produção de cana-energia $(j)$, ambos em Reais $(\mathrm{R} \$)$

As restrições descritas pelas Equações 2 e 3 garantem que a área ocupada $(X)$ por cada variedade de cana $(i, j)$ não seja superior aos percentuais máximos admitidos $(G c)$ para ocupação da área total $(A a+A e)$ em relação à cada variedade de cana a ser plantada, garantido assim a respectiva diversificação varietal.

As restrições descritas pelas Equações 4 e 5 garantem que os somatórios das áreas ocupadas $(X)$ pelas variedades de cana $(i, j)$ sejam iguais a área total destinada ao cultivo da canade-açúcar $(A a)$ e a área total destinada ao cultivo de cana-energia $(A e)$, respectivamente. Sendo que, dessa forma, a igualdade descrita nessas duas equações também garante que toda a área destinada ao plantio e cultivo da cana $(A a+A e)$ seja ocupada.

As restrições representadas pela Equações 6 e 7 garantem que as demandas $(D)$ de sacarose e de fibra, em toneladas ( $\mathrm{t})$, sejam atendidas, considerando as variedades de cana $(i, j)$ analisadas para plantio, bem como, as respectivas produtividades (Prod) estimadas pelos seus fornecedores de mudas.

A restrição descrita pela Equação 8 garante que os valores a serem atribuídos às variáveis de decisão do modelo $(X)$ sejam maiores ou iguais a zero, pois, uma vez que se trata da área de cultivo medida em hectares, não são aceitos valores negativos.

A implementação computacional realizada para o teste e validação do modelo proposto nesta investigação foi realizada mediante a simulação de três níveis de diversificação varietal ou graus de concentração varietal ( $G c=15,00 \% ; G c=12,50 \% ; G c=10,00 \%)$ em três cenários formados por três diferente composições proporções de ocupação da área total destinada ao plantio ( $A a=217$ ha e $A e=109$ ha; $A a=163$ ha e $A e=163$ ha; $A a=109$ ha e $A e=217$ ha), cujos 
parâmetros tomaram por base as informações disponíveis na literatura e/ou tiveram seus valores gerados aleatoriamente, de forma a reproduzir situações reais de empreendimentos agrícolas produtores de cana-de-açúcar e cana-energia da região centro-sul do Brasil.

A implementação e resolução do PPL ocorreu mediante o uso do pacote analítico SOLVER, disponível em planilhas eletrônicas de cálculo e, ainda, de um computador com processador Intel Core $\circledR^{\circledR}$ i5 2,30 GHz com 8,00 GB de memória RAM instalada.

\section{TESTES COMPUTACIONAIS E ANÁLISE DOS RESULTADOS}

Nesta seção, inicialmente, será descrito o processo de composição da base de dados utilizada para a implementação computacional realizada para teste e validação do modelo proposto. Na sequência, serão descritos e analisados os resultados do processo de implementação computacional para teste e validação do modelo em questão.

\subsection{Composição da Base de Dados para Realização dos Testes Computacionais}

Além dos três níveis de diversificação varietal ( $G c=15,00 \% ; G c=12,50 \% ; G c=10,00 \%)$, em 3 cenários formados por diferentes combinações de distribuição/proporção da área de plantio ( $A a=217$ e $A e=109 ; A a=163$ e $A e=163 ; A a=109$ e $A e=217$ ), a composição da base de dados dos testes computacionais realizados para validação do modelo de otimização proposto levou em conta os parâmetros descritos no Quadro 2, e ainda, uma demanda de sacarose para toda a safra $\left(D_{\text {sacarose }}\right)$ de $7.612,81 \mathrm{t}$ e uma demanda de fibra para toda a safra $\left(D_{\text {fibra }}\right)$ de $6.920,73 \mathrm{t}$.

Quadro 2 - Variedades de cana-de-açúcar e cana-energia e seus parâmetros técnico-produtivos

\begin{tabular}{|c|c|c|c|c|c|}
\hline$i$ & Variedade & $\begin{array}{c}\text { Produtividade de cana } \\
\text { (Prod) } \\
\mathbf{t ~ h a}^{-1} \\
\end{array}$ & $\begin{array}{c}\text { Sacarose } \\
(\mathrm{Pol}) \\
\%\end{array}$ & $\begin{array}{c}\text { Fibra } \\
(\text { Fib }) \\
\%\end{array}$ & $\begin{array}{c}\text { Custo } \\
(C) \\
\mathbf{R} \$ \mathbf{h a}^{-1}\end{array}$ \\
\hline 1 & CTC15 & 132,80 & 14,50 & 12,36 & $5.844,00$ \\
\hline 2 & CTC9 & 100,00 & 15,84 & 12,34 & $5.927,00$ \\
\hline 3 & RB925211 & 89,29 & 14,67 & 12,30 & $6.248,00$ \\
\hline 4 & CTC6 & 136,00 & 14,98 & 11,16 & $5.757,00$ \\
\hline 5 & RB855156 & 117,80 & 14,50 & 12,41 & $6.265,00$ \\
\hline 6 & CTC2 & 129,10 & 14,31 & 12,21 & $5.883,00$ \\
\hline 7 & RB857515 & 148,20 & 14,82 & 11,47 & $4.904,00$ \\
\hline 8 & SP80-1842 & 112,80 & 14,90 & 12,90 & $5.012,00$ \\
\hline 9 & \begin{tabular}{|l|} 
SP83-2847 \\
\end{tabular} & 126,70 & 13,20 & 12,74 & $5.948,00$ \\
\hline 10 & SP80-3280 & 121,70 & 14,80 & 11,30 & $5.673,00$ \\
\hline 11 & RB928062 & 113,00 & 15,75 & 12,38 & $5.383,00$ \\
\hline 12 & RB966928 & 123,10 & 13,32 & 11,97 & $6.338,00$ \\
\hline 13 & CTC20 & 165,00 & 13,50 & 11,50 & $4.993,00$ \\
\hline 14 & CTC17 & 112,30 & 14,98 & 12,38 & $5.460,00$ \\
\hline 15 & \begin{tabular}{|l|l|} 
SP81-3250 \\
\end{tabular} & 140,60 & 15,02 & 12,91 & $6.680,00$ \\
\hline 16 & CTC4 & 130,90 & 13,54 & 11,80 & $5.306,00$ \\
\hline 17 & RB92579 & 142,40 & 15,70 & 12,93 & $6.046,00$ \\
\hline 18 & RB855453 & 133,35 & 13,90 & 12,38 & $5.365,00$ \\
\hline
\end{tabular}


Quadro 2 - Variedades de cana-de-açúcar e cana-energia e seus parâmetros técnico-produtivos

\begin{tabular}{|c|c|c|c|c|c|}
\hline$j$ & Variedade & $\begin{array}{c}\text { Produtividade de cana } \\
\text { (Prod) } \\
\text { t ha }^{-1}\end{array}$ & $\begin{array}{c}\text { Sacarose } \\
(P o l) \\
\%\end{array}$ & $\begin{array}{c}\text { Fibra } \\
(\text { Fib }) \\
\%\end{array}$ & $\begin{array}{c}\text { Custo } \\
(C) \\
\mathbf{R} \$ \mathbf{h a}^{-1}\end{array}$ \\
\hline 1 & Cenerg-1 & 364,65 & 11,50 & 16,47 & $6.755,00$ \\
\hline 2 & Cenerg-2 & 327,52 & 12,62 & 16,43 & $5.878,00$ \\
\hline 3 & Cenerg-3 & 314,70 & 13,37 & 18,52 & $6.316,00$ \\
\hline 4 & Cenerg-4 & 310,73 & 12,79 & 18,49 & $4.965,00$ \\
\hline
\end{tabular}

Fonte: elaborado pelo autor, a partir dos dados de Ramos (2010), Nervis (2015) e Caversan (2017).

Em relação aos parâmetros técnico-produtivos descritos no Quadro 2, cabe destacar que as informações referentes à produtividade (Prod), sacarose $(P o l)$ e fibra $(F i b)$ das variedades de cana-de-açúcar ( $i$ ) estão integralmente disponíveis na pesquisa elaborada por Nervis (2015). Contudo, devido à ausência de informações técnicas, esses mesmos parâmetros relacionados à cana-energia ( $j$ ) foram estimados com base na pesquisa de Caversan (2017), nas seguintes proporções:

a)produtividade (prod) de cana-energia $\left(\mathrm{t} \mathrm{ha}^{-1}\right): 2,21$ vezes a produtividade $\left(\mathrm{t} \mathrm{ha}^{-1}\right)$ das 4 variedades com maiores produtividades de cana-de-açúcar (da maior para a menor);

b)Pol (\%): 0,8518 vezes o percentual de Pol das 4 variedade com maiores produtividades de cana-de-açúcar (da maior para a menor); e

c)fibra (\%): 1,432 vezes o percentual de Pol das 4 variedade com maiores produtividades de cana-de-açúcar (da maior para a menor);

Em relação ao custo por hectare de cana $\left(\mathrm{R} \$ \mathrm{ha}^{-1}\right)$ do plantio até colheita, com coleta de palhiço, a partir dos dados da pesquisa de Ramos (2010), foram estimados a valores aleatórios entre $\mathrm{R} \$ 4.844,00\left(\mathrm{R} \$ 5.844,00 . \mathrm{ha}^{-1}\right.$ - \$1.000,00.ha $\left.{ }^{-1}\right)$ e $\mathrm{R} \$ 6.844,00\left(\mathrm{R} \$ 5.844,00 . \mathrm{ha}^{-1}+\right.$ $\left.\mathrm{R} \$ 1.000,00 \cdot \mathrm{ha}^{-1}\right)$ para cada uma das variedades de cultivares de cana $(i, j)$. Pois, uma vez que o custo médio $\left(\mathrm{R} \$ \mathrm{ha}^{-1}\right.$ ) apurado por Ramos (2010) foi de $\mathrm{R} \$ 5.844 .00 \mathrm{ha}^{-1}$, arbitrou-se um intervalo de variação de $\mathrm{R} \$ 1.000,00$, pra cima e para pra baixo, como estratégia para garantir a aleatoriedade dos valores de custos, e ainda, para suprir possíveis perdas de poder aquisitivo da moeda (R\$) do ano de 2010 até o ano de 2021.

Para estimativa dos parâmetros referentes à demanda de sacarose para toda a safra $\left(D_{\text {sacarose }}=7.612,81 \mathrm{t}\right)$, levou-se em conta área total destinada ao plantio e cultivo da cana $(A a+$ $A e=326$ ha), a média da produtividade de cana (t) de todas as variedades analisadas no Quadro 2 (163,30 $\left.\mathrm{t} \mathrm{ha}^{-1}\right)$, e ainda, a média dos percentuais de Pol descritos por Cherubin (2015), Vian (2018) e Matsuoka (2017) $([12,90 \%+14,00 \%+16,00 \%] / 3=14,30 \%)$, ou seja: $7.612,81 \mathrm{t}=326$ ha. $163,30 \mathrm{t} \mathrm{ha}^{-1} \cdot 14,30 \%$.

Sendo que, a área total destinada ao plantio e cultivo da cana $(A a+A e)$ foi estimada com base nos dados do Censo Agropecuário do Instituto Brasileiro de Geografia e Estatística (IBGE) (SIDRA, 2019), segundo o qual, a área total ocupada pelos 14.910 empreendimentos agrícolas 
produtores de cana-de-açúcar no estado de São Paulo é de 4.861 .491 ha, perfazendo uma área média de 326 ha unidade produtora- ${ }^{1}$ (4.861.491 ha / 14.910 unidades produtoras).

Para estimativa dos parâmetros referentes à demanda de fibra para toda a safra $\left(D_{\text {fibra }}=\right.$ $6.920,73 \mathrm{t}$ ), levou-se em conta aquela mesma área total destinada ao plantio e cultivo da cana ( $A a$ $+A e=326 \mathrm{ha}$ ), a média da produtividade de cana $(\mathrm{t})$ de todas as variedades analisadas no Quadro $2\left(163,30 \mathrm{t} \mathrm{ha}^{-1}\right)$, e ainda, a média dos percentuais de fibra descritos por Vian (2018) e Matsuoka (2017), que foi de $13,00 \%([12,00 \%+14,00 \%] / 2)$, ou seja: $6.920,73 \mathrm{t}=326$ ha. $163,30 \mathrm{t} \mathrm{ha}{ }^{-1}$. $13,00 \%$.

\subsection{Aplicação do Modelo e Resultados}

No cenário " $A a=217$ ha e $A e=109$ ha", portanto, em uma situação em que a área para plantio de cana-de-açúcar $(A a)$ seria de $2 / 3$ da área total (326 ha . 2/3 = 217 ha) e área a ser plantada com cana-energia $(A e)$ seria de $1 / 3$ da área total $(326$ ha $.1 / 3=109$ ha), o custo total otimizado (mínimo) da safra variou de $\mathrm{R} \$ 1.704 .286,40$ (para $G c=15,00 \%$ ) até R $\$ 1.761 .277,20$ $(G c=10,00 \%$ ), passando por $\mathrm{R} \$ 1.729 .252,00$ (para $G c=12,50 \%$ ), conforme pode ser observado na Tabela 1 .

Tabela 1 - Resultados da aplicação do modelo no cenário " $A a=217$ ha e $A e=109$ ha"

\begin{tabular}{|c|c|c|c|}
\hline Parâmetros & $G c=15,00 \%$ & $G c=12,50 \%$ & $G c=10,00 \%$ \\
\hline$A a$ para $i=\operatorname{RB} 857515$ (ha) & 48,90 & 40,75 & 32,60 \\
\hline$A a$ para $i=\operatorname{SP} 80-1842(\mathrm{ha})$ & 48,90 & 40,75 & 32,60 \\
\hline$A a$ para $i=\mathrm{RB} 928062$ (ha) & 0,00 & 13,25 & 32,60 \\
\hline$A a$ para $i=$ CTC20 (ha) & 48,90 & 40,75 & 32,60 \\
\hline$A a$ para $i=$ CTC17 (ha) & 0,00 & 0,00 & 21,40 \\
\hline$A a$ para $i=$ CTC $4($ ha) & 48,90 & 40,75 & 32,60 \\
\hline$A a$ para $i=\operatorname{RB} 855453$ (ha) & 21,40 & 40,75 & 32,60 \\
\hline$A e$ para $j=$ Cenerg -1 (ha) & 0,00 & 0,00 & 11,20 \\
\hline Ae para $j=$ Cenerg -2 (ha) & 48,90 & 40,75 & 32,60 \\
\hline Ae para $j=$ Cenerg -3 (ha) & 11,20 & 27,50 & 32,60 \\
\hline$A e$ para $j=$ Cenerg- 4 (ha) & 48,90 & 40,75 & 32,60 \\
\hline Quantidade de variedades & 8 & 9 & 11 \\
\hline Produção de sacarose (t) & $8.685,89$ & $8.663,45$ & $8.598,12$ \\
\hline Produção de fibras (t) & $9.671,52$ & $9.681,85$ & $9.640,99$ \\
\hline Custo total (R\$) & $1.704 .286,40$ & $1.729 .252,00$ & $1.761 .277,20$ \\
\hline Área total para cana-de-açúcar (ha) & 217,00 & 217,00 & 217,00 \\
\hline Área total para cana-energia (ha) & 109,00 & 109,00 & 109,00 \\
\hline
\end{tabular}

Fonte: elaborado pelo autor, a partir dos dados pesquisa.

As informações referentes à implementação do modelo proposto para o cenário " $A a=217$ ha e $A e=109$ ha", resumidas na Tabela 1, demonstram que além de identificar as variedades de cana-de-açúcar e cana-energia as serem plantadas, de forma a minimizar o custo da safra, permitir atender com folga as demandas de matéria-prima (sacarose e fibra) e garantir a ocupação de toda 
área destinada ao plantio, de acordo com o tipo de cana plantada ( $A a$ e $A e)$, permitiram identificar uma informação de grande importância acerca do processo de diversificação varietal.

Ao considerar que foram simulados 3 graus de concentração varietal distintos $(G c=$ $15,00 \%, G c=12,50 \%$ e $G c=10,00 \%$ ), foi possível avaliar qual seria o custo da safra relacionado ao processo de diversificação varietal. Para tanto, bastaria dividir a variação do custo da produção pela variação do grau de concentração varietal, conforme proposto pela Equação 9.

Custo da e diversificação varietal $=\frac{\Delta \text { Custo total }}{\Delta G c}$

Dessa forma, nesse primeiro cenário, em média, tem-se um custo na ordem de $\mathrm{R} \$$ 11.398,16 para cada $1 \%$ de diversificação varietal abaixo de $15 \%$, uma vez que a variação do custo total foi de $\mathrm{R} \$ 56.990,80$ ( $\Delta$ Custo total $=1.761 .277,20-1.704 .286,40$ ) e a variação do grau de concentração varietal foi de $5 \%(\Delta G c=15 \%-10 \%)$, desprezando-se o fato de que ocorreram pequenas quedas na produção de matéria-prima (sacarose e fibra) à medida que se aumenta a diversificação varietal, ou ainda, diminui-se o grau de concentração varietal.

A explicação para tal evidência encontra-se na necessidade de se aumentar a quantidade de variedades a serem plantadas para que se diminuam os respectivos graus de concentração, conforme pode-se observar nas informações resumidas na Tabela 1.

Nesse sentido, além de indicar quais variedades plantar e qual a área máxima de plantio destinada a cada variedade a ser plantada ao longo dos talhões de unidade agrícola produtora, o modelo proposto é capaz de proporcionar informações ao gestor para suporte à tomada de decisões a partir de ponderações do tipo "custo versus benefício", uma vez que é possível comparar o custo da diversificação varietal, graus de concentração, com os possíveis custos relacionados à perdas decorrentes da ocorrência de pragas ou doenças capazes de atingir o canavial.

Valendo lembrar que, além da identificação das variedades a serem plantas e das respectivas áreas máximas destinadas ao plantio, e ainda, além do fornecimento de informações para tomada de decisão relacionada aos níveis de diversificação varietal e seus custos, o modelo proposto por este estudo científico mostrou-se capaz de fornecer também informações acerca de variáveis como produção de sacarose esperada ou prevista (t), produção de fibras esperada ou prevista (t), bem como, a respectiva previsão do custo total da safra $(\mathrm{R} \$)$.

Ao aplicar o modelo proposto no cenário " $A a=163$ ha e $A e=163$ ha", portanto, em uma situação em que a área a ser plantada com cana-de-açúcar $(A a)$ seria de 1/2 da área total (326 ha . $1 / 2=163$ ha) e área a ser plantada com cana-energia $(A e)$ seria também de 1/2 da área total (326 
ha $\cdot 1 / 2=163$ ha), o custo total otimizado (mínimo) da safra variou de $\mathrm{R} \$ 1.764 .719,50$ até $\mathrm{R} \$$ 1.798.256,75, segundo aquelas dois primeiros graus máximos de concentração varietal $(G c=15,00 \%$ e $G c=12,50 \%)$ estabelecidos para este experimento computacional, conforme pode ser observado na Tabela 2 .

Em relação ao menor grau de concentração $(G c=10,00 \%)$, o modelo proposto identificou que não seria possível satisfazer à restrição descrita pela Equação 5. Isso é, a maior diversificação varietal imposta por um grau máximo de concentração $(G c)$ de $10 \%$ demandaria mais do que 4 variedades de cana-energia para que a respectiva área de plantio (Ae) totalizasse 163 ha, uma vez que, com apenas 4 variedades, a área de plantio máxima de cana-energia (Ae) seria de 134,4 ha, portanto, 130,40 ha $<163,00$ ha.

Tabela 2 - Resultados da aplicação do modelo no cenário " $A a=163$ ha e $A e=163$ ha"

\begin{tabular}{lrrr}
\hline Parâmetros & $G c=15,00 \%$ & $G c=12,50 \%$ & $G c=10,00 \%$ \\
\hline A $a$ para $i=$ CTC6 & 0,00 & 0,00 & 32,60 \\
A $a$ para $i=$ RB857515 & 48,90 & 40,75 & 32,60 \\
A $a$ para $i=$ SP80-1842 & 48,90 & 40,75 & 0,00 \\
A $a$ para $i=$ SP80-3280 & 0,00 & 0,00 & 32,60 \\
Aa para $i=$ RB928062 & 0,00 & 0,00 & 32,60 \\
Aa para $i=$ CTC20 & 48,90 & 40,75 & 0,00 \\
A $a$ para $i=$ CTC4 & 16,30 & 40,75 & 0,00 \\
Aa para $i=$ RB92579 & 0,00 & 0,00 & 32,60 \\
Ae para $j=$ Cenerg-1 & 16,30 & 40,75 & 32,60 \\
Ae para $j=$ Cenerg-2 & 48,90 & 40,75 & 32,60 \\
Ae para $j=$ Cenerg-3 & 48,90 & 40,75 & 32,60 \\
Ae para $j=$ Cenerg-4 & 48,90 & 40,75 & 32,60 \\
\hline Quantidade de variedades & 8 & 8 & 10 \\
\hline Produção de sacarose (t) & $9.981,71$ & $9.938,82$ & $8.659,54$ \\
\hline Produção de fibras (t) & $11.990,28$ & $12.042,90$ & $10.037,22$ \\
\hline Custo total $(\mathrm{R} \$$ ) & $1.764 .719,50$ & $1.798 .256,75$ & $1.684 .670,20$ \\
\hline Área total para cana-de-açúcar (ha) & 163,00 & 163,00 & 163,00 \\
\hline Área total para cana-energia (ha) & 163,00 & 163,00 & 130,40 \\
\hline
\end{tabular}

Fonte: elaborado pelo autor, a partir dos dados pesquisa.

Contudo, ainda assim é possível conhecer o custo da diversificação varietal neste cenário, pois, conforme pode ser observado nas informações descritas na Tabela 2, o fato de se reduzir as áreas de plantio ( $A a$ e $A e)$ de todas aquelas variedades de cana $(i, j)$ de 48,90 ha $(G c=15 \%)$ para áreas de plantio $(A a$ e $A e)$ de 40,75 ha $(G c=12,5 \%)$ fez com que o custo total da safra se elevasse em $\mathrm{R} \$ 33.537,25(1.798 .256,75$-1.764.719,50), diante de uma redução de concentração varietal de 2,5\% $(\Delta G c=15,00 \%$ - 12,50\%), perfazendo um custo unitário de $\mathrm{R} \$ 13.414,90$ para cada $1 \%$ de diversificação varietal abaixo de $15 \%$.

A explicação para tal evidência está no fato de que se aumentou a área de plantio de uma variedade de cana-energia (Cenerg-1) com um custo ha ${ }^{-1}$ relativamente maior que as demais variedades de cana-energia, uma vez que não houve alteração na quantidade de variedades 
identificadas para plantio mesmo diante de uma variação de $2,5 \%$ no grau de concentração varietal, conforme pode-se observar nas informações resumidas na Tabela 2.

Adicionalmente deve-se destacar que o fato da aplicação do modelo proposto não permitir satisfazer à restrição descrita pela Equação 5 quando o grau de concentração varietal foi de $10 \%$ ( $G c=10,00 \%)$, no cenário " $A a=163$ ha e $A e=163$ ha", constitui-se em mais uma evidência da sua capacidade informativa. Pois, o modelo foi capaz de sinalizar que, para se atingir um grau de concentração de $10 \%$ no cenário em questão, seria preciso ampliar a quantidade de variedades de cana-energia (diversificar mais), uma vez que, em caso contrário, incorrer-se-ia em um nível de concentração varietal superior àquele proposto para o cenário em questão.

Essa evidência acerca da capacidade informativa do modelo proposto ao ferir a restrição descrita pela Equação 5, portanto, em relação à possibilidade de concentral varietal indesejada para um cenário de maior proporção de área de plantio destinada à cana-energia $(A e)$, pôde ser corroborada a partir da aplicação computacional do modelo proposto no cenário " $A a=109$ ha e $A e=217$ ha", conforme demonstram as informações detalhadas pela Tabela 3.

Tabela 3 - Resultados da aplicação do modelo no cenário " $A a=109$ ha e $A e=217$ ha"

\begin{tabular}{|c|c|c|c|}
\hline Parâmetros & $G c=15,00 \%$ & $G c=12,50 \%$ & $G c=10,00 \%$ \\
\hline Aa para $i=$ CTC9 & 11,20 & 40,75 & 0,00 \\
\hline$A a$ para $i=\mathrm{RB} 925211$ & 48,90 & 0,00 & 0,00 \\
\hline A $a$ para $i=$ CTC6 & 0,00 & 27,50 & 32,60 \\
\hline$A a$ para $i=\mathrm{RB} 857515$ & 48,90 & 0,00 & 32,60 \\
\hline$A a$ para $i=\mathrm{SP} 80-3280$ & 0,00 & 40,75 & 11,20 \\
\hline A $a$ para $i=\mathrm{RB} 92579$ & 0,00 & 0,00 & 32,60 \\
\hline Ae para $j=$ Cenerg- 1 & 48,90 & 40,75 & 32,60 \\
\hline Ae para $j=$ Cenerg -2 & 48,90 & 40,75 & 32,60 \\
\hline Ae para $j=$ Cenerg -3 & 48,90 & 40,75 & 32,60 \\
\hline Ae para $j=$ Cenerg -4 & 48,90 & 40,75 & 32,60 \\
\hline Quantidade de variedades & 7 & 7 & 8 \\
\hline Produção de sacarose (t) & $9.966,71$ & $8.668,68$ & $7.693,89$ \\
\hline Produção de fibras $(\mathrm{t})$ & $12.731,99$ & $10.835,22$ & $9.286,87$ \\
\hline Custo total $(\mathrm{R} \$)$ & $1.781 .109,80$ & $1.605 .513,00$ & $1.387 .782,20$ \\
\hline Área total para cana-de-açúcar (ha) & 109,00 & 109,00 & 109,00 \\
\hline Área total para cana-energia (ha) & 195,60 & 163,00 & 130,40 \\
\hline
\end{tabular}

Fonte: elaborado pelo autor, a partir dos dados pesquisa.

Assim, segundo as informações apresentadas pela Tabela 3, o modelo proposto foi eficiente em garantir o atendimento da demanda de matéria-prima (sacarose e fibra produzidas), minimizou os custos até onde foi possível, e ainda, garantiu que a área destinada ao plantio de cana-de-açúcar fosse totalmente ocupada. Contudo, uma vez que o cenário " $A a=109$ ha e $A e=217$ ha" demanda que área total para plantio de cana-energia seja de 217 ha ( $A e=217$ ha), o modelo proposto indicou que isso não seria possível com apenas 4 variedades de cana-energia, revelando a necessidade de ampliação da quantidade variedades desse tipo de cana para plantio e, por consequência, a necessidade de maior diversificação varietal. 


\section{CONSIDERAÇÕES FINAIS}

O modelo de otimização proposto a partir desta investigação científica destinou-se ao suporte de informações voltadas para tomada de decisões relacionadas à escolha de variedades cana-de-açúcar e cana-energia para plantio, mediante a identificação da área máxima a ser ocupada por cada variedade de cultivar, com a principal finalidade de minimizar os custos de produção de toda a safra, contudo, sem perder de vistas o atendimento aos parâmetros técnicoprodutivos relacionadas à demanda por matéria-prima, à ocupação integral das respectivas áreas de plantio, e ainda, à promoção da diversificação varietal.

Nesse sentido, o modelo em questão mostrou-se eficiente não só no cumprimento de suas premissas básicas, ou seja, o pressuposto da otimização voltada para a minimização de custos e cumprimento das restrições que lhe foram impostas, mas, também mostrou-se especialmente eficiente ao proporcionar informações capazes de permitir apurar o custo do processo de diversificação varietal propriamente dito.

Como principal limitação deste estudo, observa-se a necessidade de se conhecer, pelo menos de forma estimativa, os custos relacionados ao processo de cultivo das variedades de canade-açúcar e cana-energia consideradas no processo de planejamento. Porém, cabe destacar que tal limitação pode-se superada a partir da consulta à ampla literatura disponível sobre essa temática, sem que haja necessidade de dispêndios de tempo e dinheiro para a realização de experimentos de campo.

Para a continuidade desta pesquisa, recomenda-se a aplicação da modelagem matemática de otimização ora proposta mediante a utilização de maiores quantidades de variedades de canaenergia, e ainda, a introdução de parâmetros relacionados à existência de talhões já demarcados dentro da unidade agrícola produtora de cana.

Assim, vislumbra-se a possibilidade que os resultados relatados possam ser somados aos resultados de outros estudos científicos de natureza correlata e, por consequência, se possa ampliar o conhecimento relacionado à aplicação de modelagens matemáticas de otimização na cultura da cana-de-açúcar e da cana-energia.

\section{REFERÊNCIAS}

ANSARI, S. L., BELL, J. E.. Target Costing: the next frontier in strategic cost management. Chicago-IL: Irwin Professional Publishing, 1997.

ARENALES, M.; ARMENTANO, V.; MORABITO, R.; YANASSE, H.. Pesquisa operacional. 2. ed. Rio de Janeiro: Elsevier, 2015. 
BRAGA JUNIOR, R. L. do C.; LANDELL, M. G. de A.; SILVA, D. N. da; BIDÓIA, M. A. P.; SILVA, T. N. da; THOMAZINHO JÚNIOR, J. R.; SILVA, V. H. Pa. da. Censo varietal IAC de Cana-de-açúcar na região centro-sul do Brasil: safra 2016/17. Boletim Técnico, 217, IAC, 2017. Campinas: Instituto Agronômico, 2017. Disponível em: http://www.iac.sp.gov.br/publicacoes/porassunto/pdf/Boletim217.pdf. Acesso em: 22 maio 2020.

BRASIL. Boletim Mensal dos Biocombustíveis: divulgação dos resultados do censo varietal IAC 2016. ed. 105. Brasília: Ministério de Minas e Energia, Secretaria de Petróleo, Gás Natural e Biocombustíveis, Departamento de Biocombustíveis, nov. 2016. Disponível em: http://www.mme.gov.br/documents/1138769/1732805/Boletim+DBio+n\%C2\%BA+105+novemb ro+de+2016.doc/5ffe2880-3d0b-437c-b754-aabd8356c8e1. Acesso em: 22 maio 2020.

CAIXETA-FILHO, J. V. ; MIYASHITA, A. E.. Continuous periods for harvesting schedules: a numerical application for the brazilian sugarcane industry. Pesqui. Oper., Rio de Janeiro-RJ, v.38, n.3, p. 535-554, sept./dec. 2018. Disponível em: http://dx.doi.org/10.1590/01017438.2018.038.03.0535. Acesso em: 05 fev. 2020.

CARMO, C. R. S.. Modelo matemático de otimização para a seleção de agentes indutores de maturação aplicados na cultura da cana-de-açúcar. Nucleus, Ituverava, v.17, n.2, p. 257-277, out.2020. Disponível em: https://doi.org/10.3738/1982.2278.3839. Acesso em: 26 fev. 2021.

CAVERSAN, A. S.. Modelo matemático para planejamento do plantio e colheita da canade-açúcar e da cana-energia. 2017. 57 f. Dissertação (Mestrado) - Faculdade de Engenharia, Universidade Estadual Paulista, Bauru, 2017. Disponível em:

https://repositorio.unesp.br/bitstream/handle/11449/152364/caversan_as_me_bauru.pdf?sequence =3\&isAllowed=y. Acesso em: 17 abr. 2019.

CHERUBIN, N.. Colheita mecanizada, geadas e manejo inadequado de variedades são alguns dos fatores que vêm contribuindo para a queda dos açúcares totais recuperáveis ao longo das últimas safras. RPA news: cana \& indústria, Ribeirão Preto, n. 186, 2015. Disponível em: http://revistarpanews.com.br/ed/71-edicao2015/edicao-186/1578-por-que-o-atr-vem-caindo. Acesso em: 28 abr. 2019.

COOPER, R.; SLAGMULDER, R.. Targeting costing and value engineering. Strategies in Confrontational Cost Management Series. Montvale-NJ: Instiitute Of Management Accountants (IMA), 1997.

DEJENIE, T. W.. Role of crop modeling in scenario development analysis and developing strategic recommendation: a review. International Journal of Scientific Research and Management (IJSRM), [S. l.], v. 7, n. 1, p. 204-212, 2019. Disponível em: https://www.ijsrm.info/index.php/ijsrm/article/view/1924/1701. Acesso em: 01 fev. 2021.

DORIGUEL, F.; CRUSCIOL, C. A. C.; FLORENTINO, H. de O.. Mathematical optimization models in the sugarcane harvesting process. In: Sugarcane: technology and research. [S. $l]$ : IntechOpen, 2017. p. 207-222 . Disponível em: http://dx.doi.org/10.5772/intechopen.71530. Acesso em: 22 fev. 2021.

FLORENTINO, H. de O.; IRAWAN, C.; ALIANO, A. F.; JONES, D. F.; CANTANE, D. R.; NERVIS, J. J.. A multiple objective methodology for sugarcane harvest management with varying maturation periods. Operations Research \& Decision Theory (Springer Science \& Business Media, LLC), [S. l.], n. 267 p. 153-177, aug. 2018. Disponível em: https://doi.org/10.1007/s10479-017-2568-2. Acesso em: 22 jan. 2021. 
ISLER, P. R.; RAMOS, R. P. ; FLORENTINO, H. de O.; CANTANE, D. R.; . Otimização aplicada ao planejamento de plantio e colheita da cana-de-açúcar utilizando graus-dias. In: SIMPÓSIO BRASILEIRO DE PESQUISA OPERACIONAL, 46., 2014, Salvador. Anais... Salvador: Sociedade Brasileira de Pesquisa Operacional (SOBRAPO), 2017. p. 757-767. Disponível em: http://www.din.uem.br/sbpo/sbpo2014/pdf/arq0218.pdf. Acesso em: 15 jun. 2019.

LORIZOLA, G. M.; CAPITANI, D. H. D.. Análise do modelo estrutura-conduta-desempenho do setor sucroenergértico brasileiro. Nucleus, Ituverava, v.15, n.2, p.383-399, out. 2018. Disponível em: http://dx.doi.org/10.3738/1982.2278.2920. Acesso em: 01 jan. 2021.

MARTYNIUK, T.; ZABLOCKA, S. Practice application of target costing case study: cost of roof production. In: CONGRESSO DA EAA (EUROPEAN ACCOUNTING ASSOCIATION), 21, 1998. Anais... Antuérpia (Bélgica): [s.n.], 1998.

MATSUOKA, S.. Teor de fibra é a trava da produtividade da cana. Revista Opiniões, Ribeirão Preto, ano 14, n. 52, divisão C, abr./jun. de 2017. Disponível em:

http://sucroenergetico.revistaopinioes.com.br/revista/detalhes/6-teor-de-fibra-e-trava-daprodutividade-da-cana/. Acesso em 28 abr. 2019.

NERVIS, J. J.. Simulação para a otimização da colheita da cana-de-açúcar. 2015. 67 f. Tese (Doutorado) - Faculdade de Ciências Agronômicas, Universidade Estadual Paulista, Botucatu, 2015. Disponível em:

https://repositorio.unesp.br/bitstream/handle/11449/139399/000865102.pdf? sequence=1\&isAllo wed=y. Acesso em: 17 abr. 2019.

NERVIS, J. J.; FLORENTINO, H. de O.; CANTANE, D. R.; RAMOS, R. P.; ISLER, P. R.. Simulação para a otimização da colheita da cana-de-açúcar. Revista Brasileira de Biometria, [S. 1.], v. 35, n. 2, p. 415-439, jun. 2017. Disponível em:

http://www.biometria.ufla.br/index.php/BBJ/article/view/47. Acesso em: 24 out. 2018.

PATCHARAMETHANON, R.; SUDTACHAT, K.. District model with two types of vehicles for transporting sugarcane. In: INTERNATIONAL CONFERENCE ON INDUSTRIAL

ENGINEERING AND APPLICATIONS, 5., 2018, Singapore. CONFERENCES... Singapore: Institute of Electrical and Electronics Engineers Singapore, 2018, p. 468-474. Disponível em: https://ieeexplore.ieee.org/document/8387146. Acesso em: 04 jan. 2021.

RAIZER, A. J.; BRAGA JÚNIOR, R. L. do C.; SELEGATO, S. M.. Índices de eficiência no uso de variedades de cana-de-açúcar. RPA News: cana \& indústria, Ribeirão Preto, ano 12, n. 151/152, p. 25-29, jul./ago. 2013. Disponível em: http://revistarpanews.com.br/edicoes/151/IDEANews151e152FinalGraficaWeb.pdf. Acesso em: 22 maio 2019.

RAMOS, R. P.. Modelo matemático para custo e energia na produção de açúcar e álcool. 2010. 72 f. Dissertação (Mestrado) - Faculdade de Ciências Agronômicas, Universidade Estadual Paulista, Botucatu, 2010. Disponível em:

https://repositorio.unesp.br/bitstream/handle/11449/90537/ramos_rp_me_botfca.pdf?sequence=1 \&isAllowed=y. Acesso em: 22 abr. 2019.

SAKURAI, M.. Gerenciamento integrado de custos. São Paulo: Atlas, 1997. 
SIDRA, Sistema IBGE de Recuperação Automática. Censo agropecuário: tabela 6615 - número de estabelecimentos agropecuários, quantidade produzida e area colhida, por produtos da lavoura temporária, resultados preliminares 2017. [S. 1.]: 2019. Disponível em:

https://biblioteca.ibge.gov.br/visualizacao/livros/liv61914.pdf. Acesso em: 26 abr. 2020.

SUNGNUL, S.; PORNPRAKUN, W.; PRASATTONG S.; BAITIANG, C.. A mathematical model for the sugarcane trading system in Thailand. Engineering Letters, [S. l.], v. 26, n. 1, p. 84-91, February 2018. Disponível em:

http://www.engineeringletters.com/issues_v26/issue 1/EL_26_1_10.pdf. Acesso em: 10 fev. 2021.

TOMAZELA, M. das G. J. M.: CAMPOS, F. C. de; DANIEL, L. A.. Mineração de dados aplicada à produtividade de cana-de-açúcar. In: ENCONTRO NACIONAL DE ENGENHARIA DE PRODUCAO, 35., 2015, Fortaleza. Anais... Fortaleza: ABEPRO, 2015. p. 2-17.

TONIELO, A. E.; PAIXÃO, A. C. S.; VANZELLA, C. A.; ORTOLAN, M. C. de A.; SICCHIERI, M. S.; BISSON, O.. Mapa da mina. Revista Canavieiros, Sertãozinho, n. 140, ano 11, p. 5-5, fev. 2018. Disponível em:

https://www.revistacanavieiros.com.br/uploads/pagina/tag/2019/09/O412EpeVUm98JBIC/09f565 a3c66e3938ab965b0e8378b66f.pdf. Acesso em: 22 fev. 2021.

UNICA, União das Indústrias de Cana-de-açúcar. Setor sucroenergético no Brasil: uma visão para 2030. São Paulo: ÚNICA, 2016. Disponível em:

http://www.mme.gov.br/documents/10584/7948692/UNICA-

CEISE_Setor+Sucroenerg\%C3\%A9tico+no+Brasil_Uma+Vis\%C3\%A3o+para+2030.pdf/80da95

80-60c7-4f53-afaf-

030ad01f3ebf;jsessionid=AC802B166C93389BED1AB445EAB7CD10.srv155. Acesso em: 21 jun. 2019.

VIAN, C. E. F.. Cana-de-Açúcar: qualidade de matéria-prima. Brasília: Agência Embrapa de Informação Tecnológica, 2018. Disponível em:

http://www.agencia.cnptia.embrapa.br/gestor/cana-de-

acucar/arvore/CONTAG01_138_22122006154842.html. Acesso em: 22 fev. 2021. 